\title{
Identification of dysregulated serum miR-508-3p and miR-885-5p as potential diagnostic biomarkers of clear cell renal carcinoma
}

\author{
SIMING LIU $^{1 *}$, XIAOJUN DENG ${ }^{1 *}$ and JIONG ZHANG ${ }^{2}$ \\ ${ }^{1}$ Department of Urology, Zhoupu Hospital Affiliated with Shanghai University of Medicine and Health Sciences; \\ ${ }^{2}$ Department of Urology, Shanghai Putuo District Liqun Hospital, Shanghai 200000, P.R. China
}

Received March 30, 2019; Accepted August 8, 2019

DOI: $10.3892 / \mathrm{mmr} .2019 .10762$

\begin{abstract}
Clear cell renal cell carcinoma (ccRCC), the most common subtype, accounts for approximately $80 \%$ of all RCC cases. ccRCC patients typically present with an advanced stage at the time of diagnosis resulting in a poor patient prognosis. The present study aimed to identify novel potential microRNAs (miRNAs or miRs) in peripheral blood as biomarkers for the detection of ccRCC. Candidate miRNAs were selected through integrated analysis of the Gene Expression Omnibus (GEO) database, The Cancer Genome Atlas (TCGA) database, and from clinical samples. The expression levels of miRNAs were quantified using reverse transcription-quantitative PCR. Receiver operating characteristic (ROC) curve analysis was used to explore the diagnostic values of the miRNAs. Bioinformatic analysis of candidate miRNAs was conducted by using the STRING database. After an integrated analysis of the GEO and TCGA databases, four miRNAs were found to be consistently dysregulated in ccRCC tissues. Then, their expression levels in serum and diagnostic utilities were further explored. We discovered that serum miR-508-3p and miR-885-5p were significantly dysregulated in ccRCC patients with marked diagnostic values. The area under the ROC curve (AUC) of serum miR-508-3p and miR-885-5p was 0.80 (95\% CI, 0.73-0.87) and 0.87 (95\% CI, 0.79-0.95), respectively. Functional enrichment analysis revealed that both miR-508-3p and miR-885-5p were closely associated with cellular metabolic processes. In conclusion, serum miR-508-3p and miR-885-5p are novel potential biomarkers for the diagnosis of ccRCC.
\end{abstract}

Correspondence to: Dr Jiong Zhang, Department of Urology, Shanghai Putuo District Liqun Hospital, 910 Taopu Road, Putuo, Shanghai 200000, P.R. China

E-mail: zhangjiongsh@163.com

*Contributed equally

Key words: clear cell renal cell carcinoma, miRNA, biomarker, serum, bioinformatic analysis

\section{Introduction}

Renal cell carcinoma (RCC), one of the most prevalent urological malignancies (1), was responsible for 175,098 tumor-related deaths worldwide in 2018 (2). Even worse, the incidence of RCC has increased in the past two decades (3). Clear cell renal cell carcinoma (ccRCC) is the most common histological and molecular subtype accounting for $\sim 80 \%$ of all RCC cases (4). Owing to the obscure symptoms, high invasiveness and high mortality associated with ccRCC, 33\% of ccRCC patients present with metastasis at the time of diagnosis, which limits the possibility of surgical intervention $(5,6)$. Thus, there is an urgent need to identify novel biomarkers for the early detection of ccRCC.

MicroRNAs (miRNAs or miRs) are a class of single-stranded noncoding RNAs (20 to 24 nucleotides in length). miRNAs can elicit either degradation or translational inhibition of target mRNAs by directly binding to the 3 '-untranslated region (3'UTR) of targets (7). Dysregulated miRNAs have been demonstrated to influence multiple processes in cancer cells, such as proliferation, migration and chemoresistance (8-10). Recently, several investigations found that differentially expressed miRNAs in cancer cells could also affect the steady abundance of stable miRNAs in the peripheral blood $(11,12)$. These findings indicate the potential role of circulating miRNAs as diagnostic biomarkers for various types of cancers. Plasma miR-21 and miR-222 were found to be markedly elevated in patients with gastric cancer and may serve as potential diagnostic biomarkers of gastric cancer (13). Furthermore, Sun et al discovered that the expression of serum miR-30a-5p was significantly downregulated in patients with colorectal cancer compared with healthy people and serum miR-30a-5p may act as a novel biomarker for the diagnosis of colorectal cancer (14). In ccRCC, despite reports that miR-144-3p, miR-126, miR-499a, miR-224 and miR-141 in peripheral blood are potential diagnostic biomarkers, these miRNAs were found to lack a high diagnostic accuracy, nor were detected in large sample sizes (15-17).

In the present study, several miRNAs were selected that were found to be consistently dysregulated in ccRCC tissues through integrated analysis of the Gene Expression Omnibus (GEO) database and The Cancer Genome Atlas (TCGA) database and their expression levels in serum samples of ccRCC patients were further explored. We identified dysregulated serum miR-508-3p and miR-885-5p as novel potential diagnostic biomarkers for ccRCC. 


\section{Materials and methods}

Study design. Our study encompassed four phases. First, the miRNA profiles in the GEO and TCGA databases were analyzed and four miRNAs consistently dysregulated in both databases were identified. Then their expression levels were tested in the serum of ccRCC patients by using a small set of samples, and their diagnostic value to differentiate ccRCC patients from healthy individuals was assessed. Next, we selected miR-508-3p and miR-885-5p whose diagnostic values were high as candidate miRNAs, and further verified their expression and diagnostic utility in an independent cohort. Finally, considering the regulatory mechanisms of miR-508-3p and miR-885-5p remained elusive, a bioinformatic analysis of both miRNAs was performed to briefly reveal their biological functions.

Enrolled participants. All participants were enrolled at the Shanghai Putuo District Liqun Hospital. A diagnosis of ccRCC was confirmed after histopathological analysis of the surgical resected tumors. None of the patients recruited in this study received chemotherapy or radiation therapy before peripheral blood collection. Age- and sex-matched healthy controls (HCs) were selected from those individuals who took part in a routine physical examination. Written informed consent was obtained from each patient and $\mathrm{HC}$ individual. This study was approved by the Research and Ethics Committee of Shanghai Putuo Liqun District Hospital.

Sample processing. After centrifugation of peripheral venous blood at $2,860 \mathrm{x}$ g for $10 \mathrm{~min}$, serum was collected and subsequently stored at $-80^{\circ} \mathrm{C}$. Total RNA was isolated from $250 \mu \mathrm{l}$ serum using traditional TRIzol ${ }^{\circledR}$ LS reagent (Invitrogen/Thermo Fisher Scientific, Inc.) according to the manufacturer's instructions. An aliquot of $1 \mu \mathrm{l}$ cel-miR-39-3p (Guangzhou RiboBio Co., Ltd.) at a concentration of $1 \mu \mathrm{M}$ was added to each sample to act as the external reference during RNA isolation.

Reverse transcription-quantitative polymerase (RT-q) PCR for miRNA quantitation. Reverse transcription and RT-qPCR for miRNAs and external reference miR-39 were conducted using a Hairpin-it microRNA RT-PCR Quantitation kit (GenePharma) according to the manufacturer's protocol. The reactions were initiated with denaturation at $95^{\circ} \mathrm{C}$ for $3 \mathrm{~min}$, followed by 40 cycles of $95^{\circ} \mathrm{C}$ for $15 \mathrm{sec}$ and $62^{\circ} \mathrm{C}$ for $34 \mathrm{sec}$. The relative expression of miRNA was calculated using the $2^{-\Delta \Delta \mathrm{Cq}}$ method (18). miRNAs with $\log \mid \mathrm{FCl}>1$ and $\mathrm{P}<0.05$ were regarded as dysregulated miRNAs.

Bioinformatic analysis. The significantly dysregulated miRNAs in the GEO or TCGA databases were calculated by using GEO2R (http://www.ncbi.nlm.nih.gov/geo/geo2r/), an online analysis tool and edgeR package in $\mathrm{R}$ language, respectively. The potential targets of miRNAs were identified by integrated analysis of the public bioinformatic algorithms TargetScan (http://www.targetscan.org/) and miRDB (mirdb. org). The functional enrichment analysis which contains molecular functions (MF), biological processes (BP) and cellular components (CC) was conducted in STRING
Table I. Clinical characteristics of the participants in the study cohort.

\begin{tabular}{lcc}
\hline Characteristics & HCs $(\mathrm{n}=35)$ & ccRCC patients $(\mathrm{n}=85)$ \\
\hline Age (years) & & 41 \\
$\leq 60$ & 17 & 44 \\
$>60$ & & \\
Sex & 19 & 45 \\
Male & 16 & 40 \\
Female & & \\
T stage & & 63 \\
T1+T2 & & 22 \\
T3+T4 & & 78 \\
N stage & & 7 \\
N0+NX & & \\
N1 & & 67 \\
M stage & 18 \\
M0+MX & \\
M1 & 46 \\
Grade & 39 \\
G1+G2 & \\
G3+G4 & & 61 \\
TNM stage & & 24 \\
I+II & \\
III+IV &
\end{tabular}

HCs, healthy controls; ccRCC, clear cell renal cell carcinoma.

(https://string-db.org/). The pathway analysis was conducted using the Kyoto Encyclopedia of Genes and Genomes (KEGG) pathway database (https://www.genome.jp/kegg/pathway. html).

Statistical analysis. Statistical analysis was performed using SPSS 22.0 (IBM Corp.) and GraphPad Prism 8.0 software (GraphPad Software, Inc.) using the Student's t-test, and Chi-square test. The receiver operating characteristic (ROC) curve was performed to evaluate the utility of miRNAs as a diagnostic biomarker of ccRCC. Cut-off values of the relative expression of miRNAs were determined using the Youden index from the ROC curves. A P-value $<0.05$ was considered to indicate statistical significance.

\section{Results}

Analysis of the dysregulated miRNAs in ccRCC based on the GEO and TCGA databases. After searching the GEO database using the keywords 'miRNA' and 'ccRCC', we found two GEO datasets [GSE109368 and GSE116251 (19)] that met our criteria. GSE109368 contains 12 paired ccRCC tissues and adjacent normal tissues (ANTs). The platform for GSE109368 analysis was the Illumina NextSeq 500 (GPL18573). GSE116251 contains 18 paired ccRCC tissues and ANTs. The platform for GSE109368 analysis was the NanoString nCounter miRNA Expression Panel (GPL25243). 

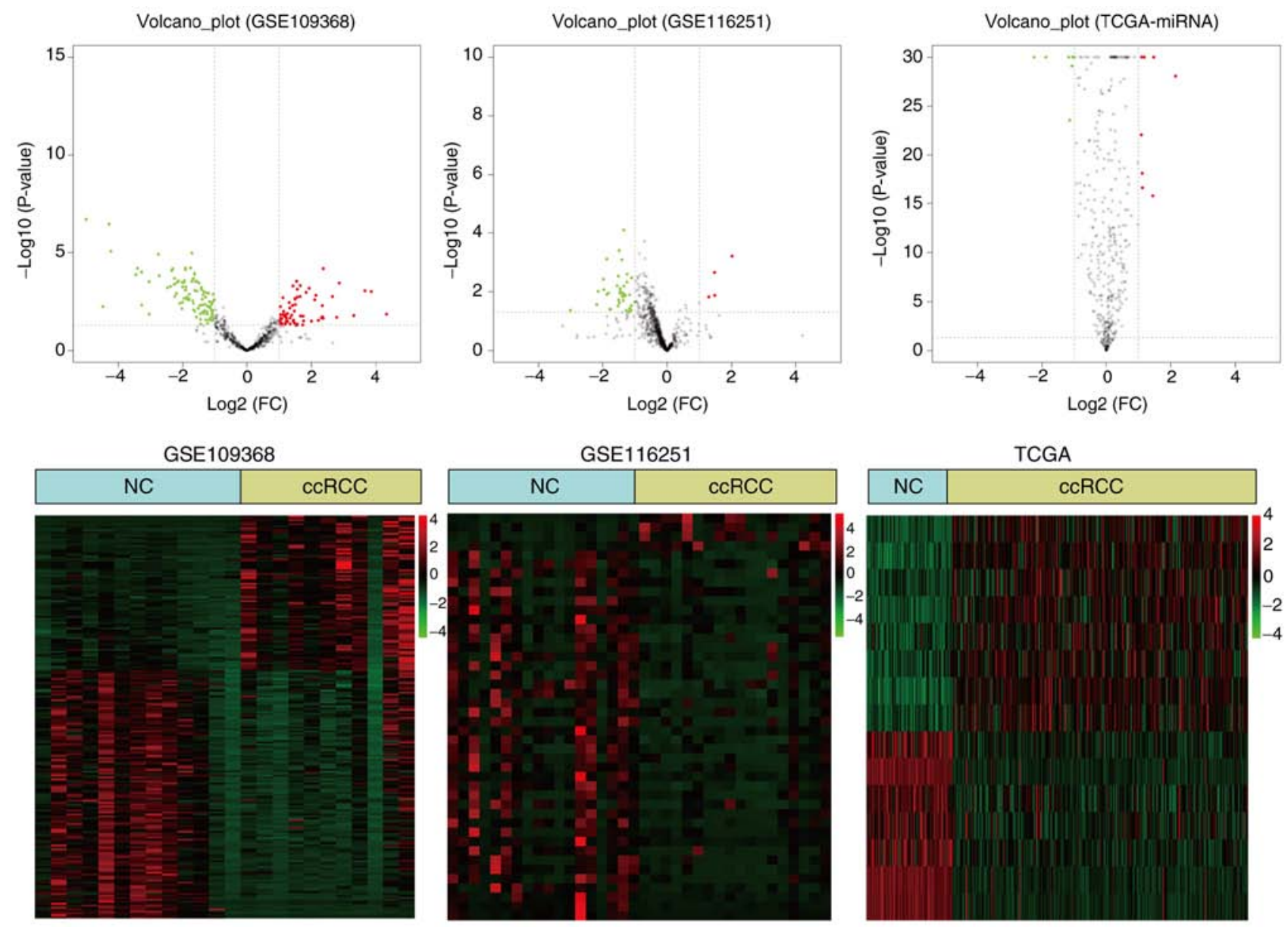

Figure 1. Analysis of dysregulated miRNAs in ccRCC based on the GEO and TCGA databases. GEO, Gene Expression Omnibus; TCGA, The Cancer Genome Atlas; NC, non-cancerous tissues; ccRCC, clear cell renal cell carcinoma tissues.

As shown in Fig. 1, 82 upregulated miRNAs and 122 downregulated miRNAs were found in GSE109368. In GSE116251, 4 upregulated miRNAs and 41 downregulated miRNAs were found. Furthermore, after analyzing the ccRCC dataset in the TCGA database, 8 upregulated miRNAs and 7 downregulated miRNAs were discovered by using R studio. Subsequently, we assembled these miRNAs to derive their intersection. It was found that miR-141-3p, miR-508-3p, miR-885-5p and miR-592 were consistently dysregulated (Fig. 2).

Differentially expressed miRNAs examined in a small set of serum samples. The expression levels of serum miR-141-3p, miR-508-3p, miR-885-5p, and miR-592 were examined in a small set of ccRCC patients $(n=10)$ and healthy controls (HCs) $(n=10)$. Serum miR-141-3p and miR-508-3p expression levels were significantly downregulated in ccRCC compared with that in the HCs. In contrast, serum miR-885-5p and miR-592 expression levels were significantly upregulated in ccRCC compared with that in the HCs (Fig. 3A). ROC analysis revealed that circulating miR-141-3p, miR-508-3p, miR-885-5p, and miR-592 could discriminate ccRCC patients from HCs with an area under the ROC curve (AUC) value of 0.73 [95\% confidence interval (CI): 0.57-0.90], 0.86 (95\% CI: 0.72-1.0), 0.91 (95\% CI: 0.82-1.0), and 0.78 (95\% CI: 0.61-0.95), respectively (Fig. 3B). Considering the markedly high diagnostic utility of miR-508-3p and miR-885-5p, we further investigated their potential to be used as diagnostic biomarkers of ccRCC subsequently.

Validating the expression levels of miR-508-3p and miR-885-5p in an independent cohort. The clinical characteristics of participants in the cohort $(n=120)$ are summarized in Table I. There were no statistically significant differences in age and sex between ccRCC patients and HCs. As shown in Fig. 4A, when compared with HCs, serum miR-508-3p levels showed a significant decrease in patients with ccRCC, while serum miR-885-5p expression was significantly elevated in ccRCC patients. ROC analysis revealed that circulating miR-508-3p and miR-885-5p could discriminate ccRCC patients from HCs with AUC values of 0.80 (95\% CI: 0.73-0.87) and 0.87 (95\% CI: 0.79-0.95), respectively (Fig. 4B). Since a combination of miRNAs could improve the diagnostic accuracy, we calculated the combination factor of serum miR-508-3p and miR-885-5p by using SPSS software and tested its diagnostic value. The combination of miR-508-3p and miR-885-5p markedly differentiated ccRCC patients from HCs with an AUC value of 0.90 (95\% CI: 0.84-0.96) (Fig. 4B). Moreover, 85 ccRCC patients were divided into a high group $(n=42)$ and a low group $(n=43)$ according to the median value of miR-508-3p or miR-885-5p expression. The Chi-square test showed that serum miR-508-3p expression was significantly correlated 
GSE109368
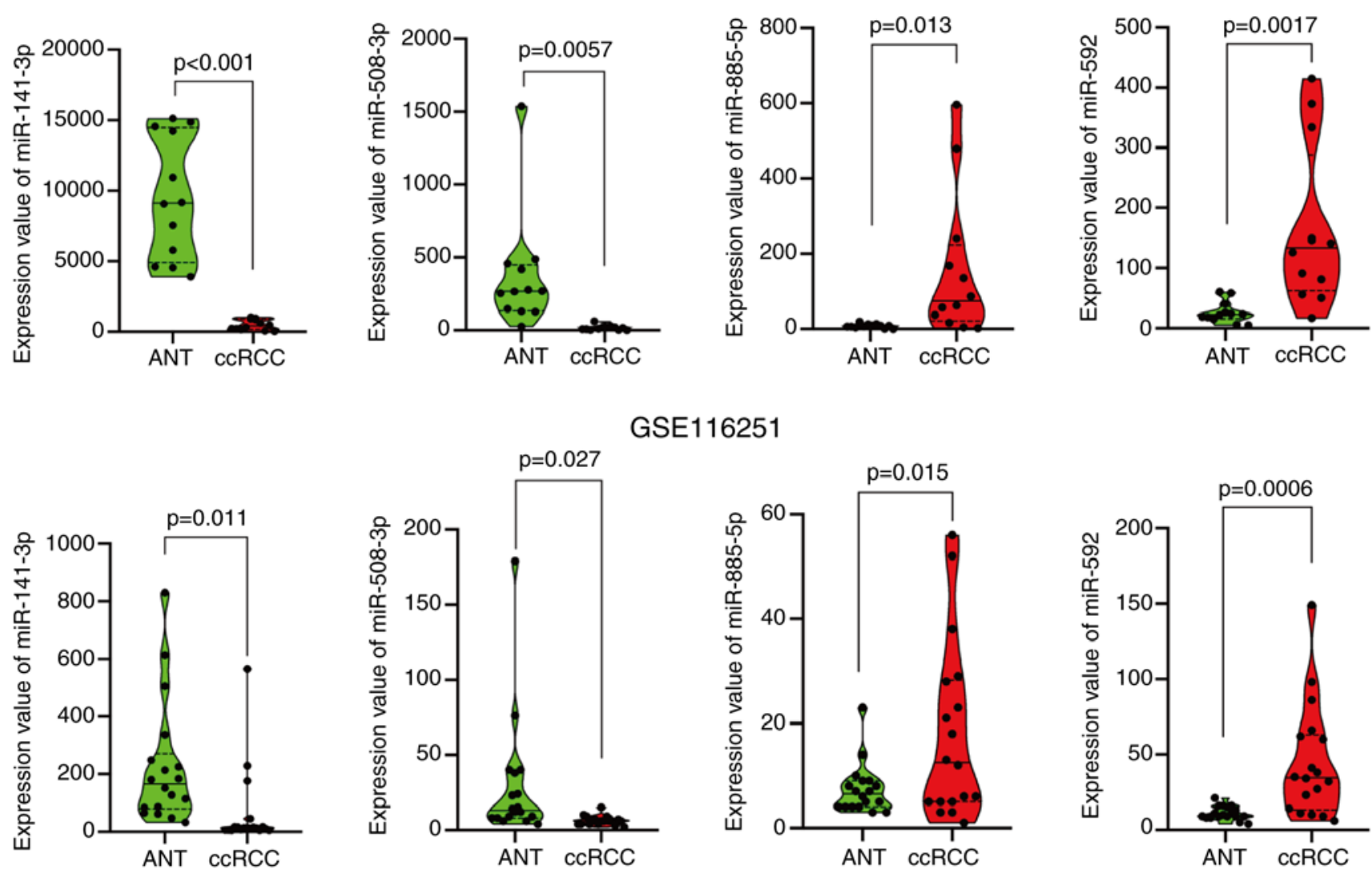

GSE116251
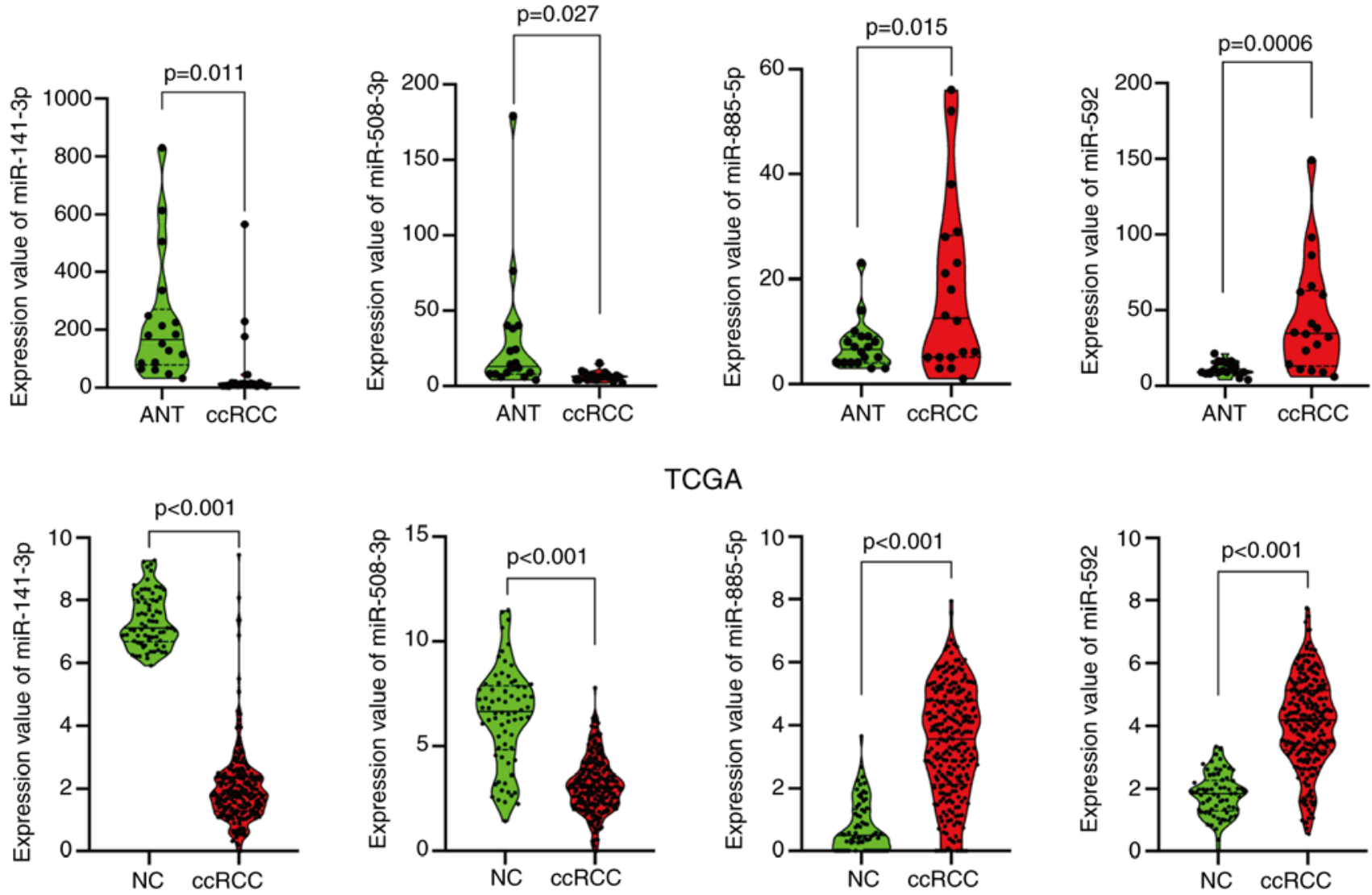

Figure 2. miRNAs consistently dysregulated in GSE109368, GSE116251 and TCGA database. TCGA, The Cancer Genome Atlas; ANT, adjacent normal tissues; ccRCC clear cell renal cell carcinoma tissues.

with $\mathrm{T}$ stage $(\mathrm{P}=0.004)$, metastasis $(\mathrm{P}=0.009)$, Fuhrman grading $(\mathrm{P}<0.001)$ and $\mathrm{TNM}$ stage $(\mathrm{P}=0.005)$; serum miR-885-5p expression was significantly correlated with $\mathrm{T}$ stage $(\mathrm{P}<0.001)$ and Fuhrman grading $(\mathrm{P}=0.013)$ (Table II).

Functional enrichment and pathway analysis of miR-508-3p and miR-885-5p. Previous analysis based on GEO and TCGA databases revealed that both miR-508-3p and miR-885-5p were significantly dysregulated in ccRCC tissues which means miR-508-3p and miR-885-5p may play important roles in the development and progression of ccRCC. Considering that the regulatory mechanisms of miR-508-3p and miR-885-5p have not been reported in ccRCC, they were analyzed using bioinformatics. As shown in Fig. 5A, 375 genes and 381 genes were identified as targets of miR-508-3p and miR-885-5p, respectively. Importantly, functional enrichment analysis showed that both targets of miR-508-3p and miR-885-5p were mainly located in the nucleus and were significantly enriched in positive regulation of metabolic processes. In the MF category, miR-508-3p and miR-885-5p were significantly associated with nucleic acid binding. We hypothesized that abnormal metabolic processing may contribute to the progression of ccRCC (Fig. 5B). KEGG analysis revealed that miR-508-3p was significantly associated with renal cell carcinoma (RCC), inositol phosphate metabolism, regulation of the actin cytoskeleton as well as pathways in cancer and that miR-885-5p may regulate ccRCC through the Hippo and Wnt signaling pathways (Fig. 5C). 
Table II. Association between serum miR-558-3p and miR-885-5p and clinical characteristics of the ccRCC patients.

\begin{tabular}{|c|c|c|c|c|c|c|}
\hline \multirow[b]{2}{*}{ Characteristics } & \multicolumn{3}{|c|}{ miR-508-3p } & \multicolumn{3}{|c|}{ miR-885-5p } \\
\hline & High & Low & P-value & High & Low & P-value \\
\hline \multicolumn{7}{|l|}{ Age (years) } \\
\hline$\leq 60$ & 22 & 19 & & 19 & 22 & \\
\hline$>60$ & 20 & 24 & 0.45 & 23 & 21 & 0.58 \\
\hline \multicolumn{7}{|l|}{ Sex } \\
\hline Male & 24 & 21 & & 22 & 23 & \\
\hline Female & 18 & 22 & 0.44 & 20 & 20 & 0.89 \\
\hline \multicolumn{7}{|l|}{ T stage } \\
\hline $\mathrm{T} 1+\mathrm{T} 2$ & 37 & 26 & & 24 & 39 & \\
\hline $\mathrm{T} 3+\mathrm{T} 4$ & 5 & 17 & 0.004 & 18 & 4 & $<0.001$ \\
\hline \multicolumn{7}{|l|}{$\mathrm{N}$ stage } \\
\hline $\mathrm{N} 0+\mathrm{NX}$ & 39 & 39 & & 37 & 41 & \\
\hline N1 & 3 & 4 & 0.71 & 5 & 2 & 0.22 \\
\hline \multicolumn{7}{|l|}{ M stage } \\
\hline $\mathrm{M} 0+\mathrm{MX}$ & 38 & 29 & & 30 & 37 & \\
\hline M1 & 4 & 14 & 0.009 & 12 & 6 & 0.086 \\
\hline \multicolumn{7}{|l|}{ Fuhrman grading } \\
\hline $\mathrm{G} 1+\mathrm{G} 2$ & 31 & 15 & & 17 & 29 & \\
\hline G3+G4 & 11 & 28 & $<0.001$ & 25 & 14 & 0.013 \\
\hline \multicolumn{7}{|l|}{ TNM stage } \\
\hline $\mathrm{I}+\mathrm{II}$ & 36 & 25 & & 27 & 34 & \\
\hline III+IV & 6 & 18 & 0.005 & 15 & 9 & 0.13 \\
\hline
\end{tabular}

ccRCC, clear cell renal cell carcinoma. P-values in bold print indicate statistically significant differences.

A

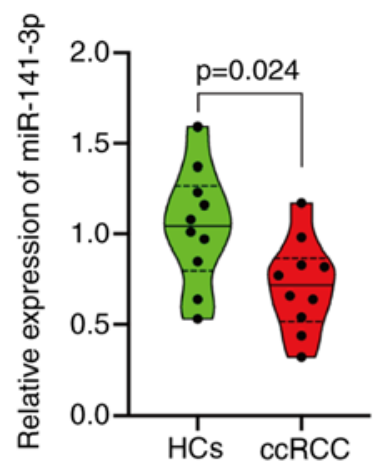

B ROC of miR-141-3p

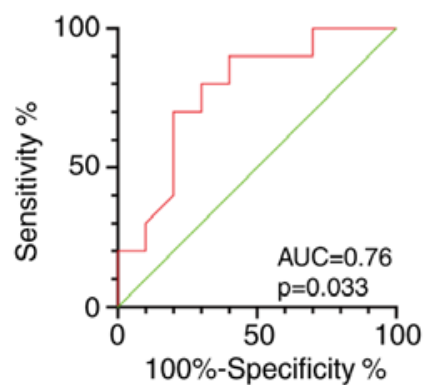

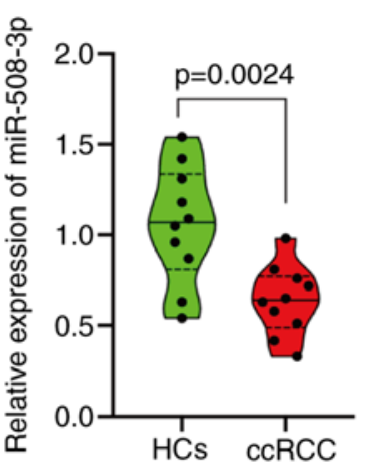

ROC of miR-508-3p

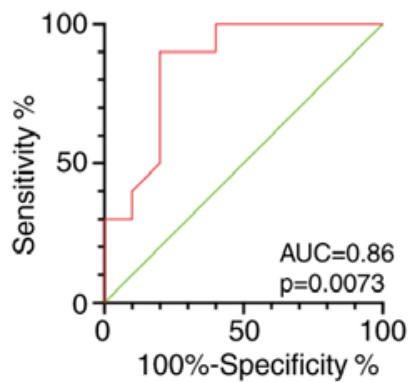

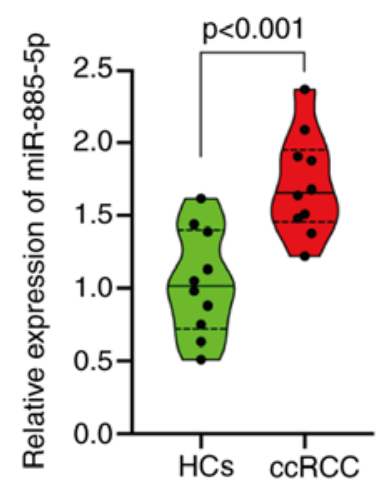

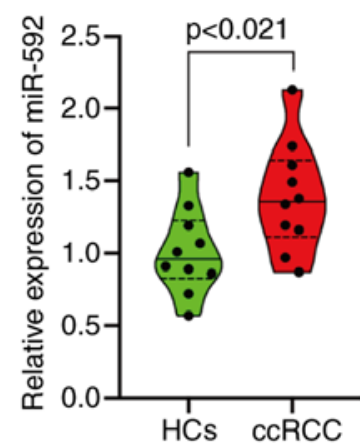

ROC of miR-885-5p

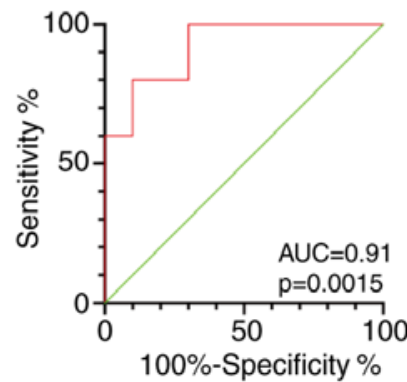

ROC of miR-592

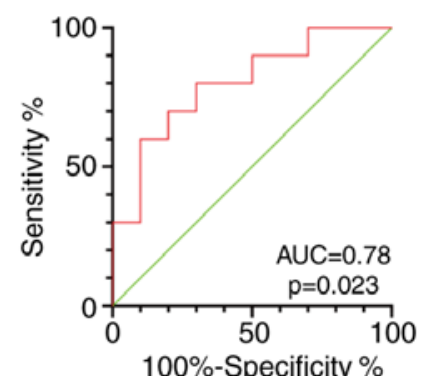

Figure 3. Differentially expressed miRNAs examined in a small set of serum samples. (A) Expression of miR-141-3p, miR-508-3p, miR-885-5p, and miR-592 in the serum of ccRCC patients compared to HCs. (B) The ROC curves of serum miR-141-3p, miR-508-3p, miR-885-5p, and miR-592 to differentiate ccRCC patients from HCs. HCs, healthy controls; ccRCC, clear cell renal cell carcinoma tissues; ROC, receiver operating characteristic; AUC, area under the ROC curve. 
A

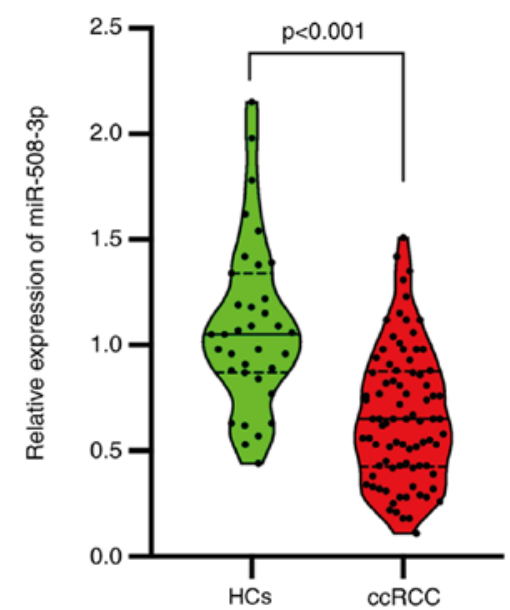

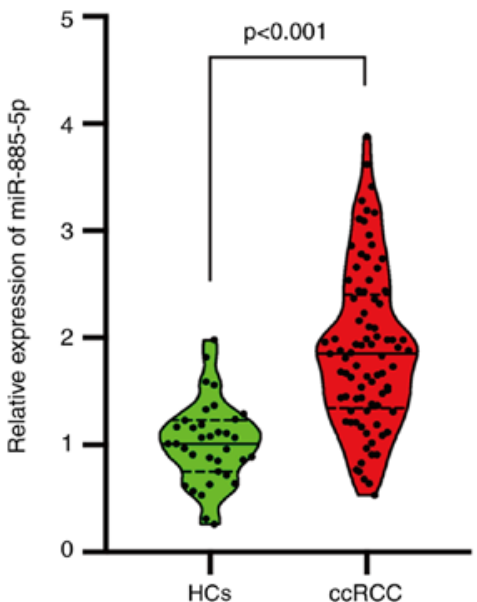

B

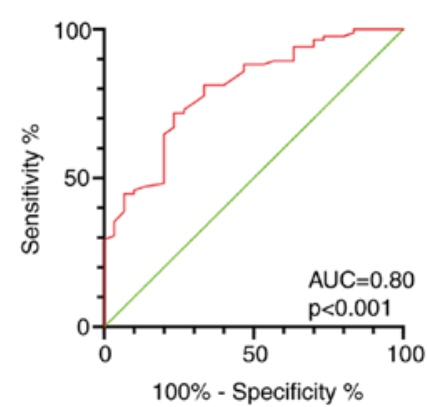

ROC of miR-885-5p

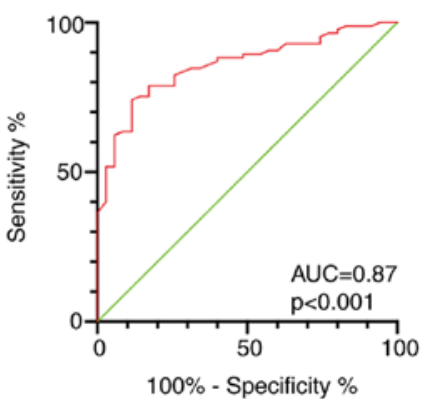

ROC of combination

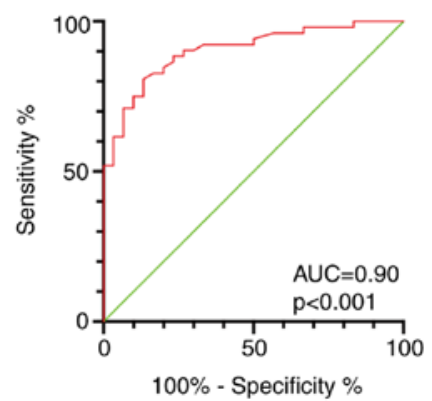

Figure 4. Validation of the expression of miR-508-3p and miR-885-5p in an independent cohort. (A) Expression of miR-508-3p and miR-885-5p in the serum of ccRCC patients compared to HCs. (B) ROC curves of serum miR-508-3p, miR-885-5p, and their combination to differentiate ccRCC patients from HCs. HCs, healthy controls; ccRCC, clear cell renal cell carcinoma tissues; ROC, receiver operating characteristic; AUC, area under the ROC curve.

\section{Discussion}

In the present study, it was discovered that serum miR-508-3p and miR-885-5p expression was significantly dysregulated in clear cell renal cell carcinoma (ccRCC) compared to that noted in the healthy controls (HCs). Serum miR-508-3p and miR-885-5p discriminated ccRCC patients from HCs with marked diagnostic utility. Moreover, bioinformatic analysis based on the GEO and TCGA databases revealed that although miR-508-5p expression was downregulated while miR-885-5p expression was upregulated in ccRCC tissues, both miRNAs may promote the development and progression of ccRCC by regulating cellular metabolic processes.

The search for abnormally expressed circulating miRNAs as diagnostic biomarkers has been widely reported for various types of cancers, including ccRCC (17,19-21). Tan et al reported that serum miR-199a expression was reduced in patients with colorectal cancer and was significantly associated with poor patient prognosis. Serum miR-199a may be used for diagnosing colorectal cancer (22). Guo and colleagues found that the serum level of miR-1915-3p was significantly upregulated and miR-455-3p was significantly downregulated in breast cancer patients compared with healthy volunteers. ROC analysis suggested that miR-1915-3p and miR-455-3p are promising serum diagnostic and predictive biomarkers of breast cancer (23). Lou et al discovered that plasma miR-144-3p was significantly upregulated in ccRCC patients serving as a promising biomarker for the detection of ccRCC (16). The present study not only provided novel potential diagnostic biomarkers of ccRCC, but also demonstrated that tumor cells could influence the expression levels of circulating miRNAs.

The abnormal expression levels of miR-508-3p and miR-885-5p have been explored in several types of cancer. Guo et al demonstrated that miR-508-3p was markedly decreased in triple-negative breast cancer. miR-508-3p was found to inhibit cell invasion and epithelial-mesenchymal transition by targeting ZEB1 in triple-negative breast cancer (24). Huang et al demonstrated that miR-508-3p concordantly silenced NFKB1 and RELA to inactivate canonical NF- $\kappa B$ signaling in gastric carcinogenesis (25). Although miR-508-3p was reported to be downregulated in the plasma of RCC patients and to act as a tumor-suppressor gene associated with the proliferation, migration, and apoptosis of RCC cells (26), its concrete diagnostic value and molecular mechanism have not been investigated. Yu et al reported that miR-885-5p, as a oncogene, promotes osteosarcoma proliferation and migration by downregulation of cell division cycle protein 73 homolog expression (27). However, the expression as well as the molecular mechanisms of miR-885-5p in ccRCC remain elusive. Our study revealed that miR-508-5p expression was downregulated, while miR-885-5p expression was upregulated in ccRCC tissues, and both miRNAs were closely associated with cellular metabolic processing for the first time. The relationship between abnormal metabolism and cancers has been investigated (28-30). KEGG pathway analysis revealed that miR-508-3p and miR-885-5p may regulate ccRCC through 
A miR-508-3p

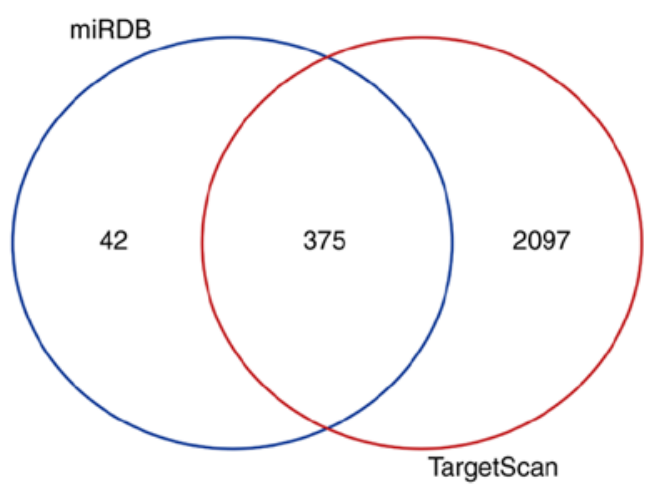

B

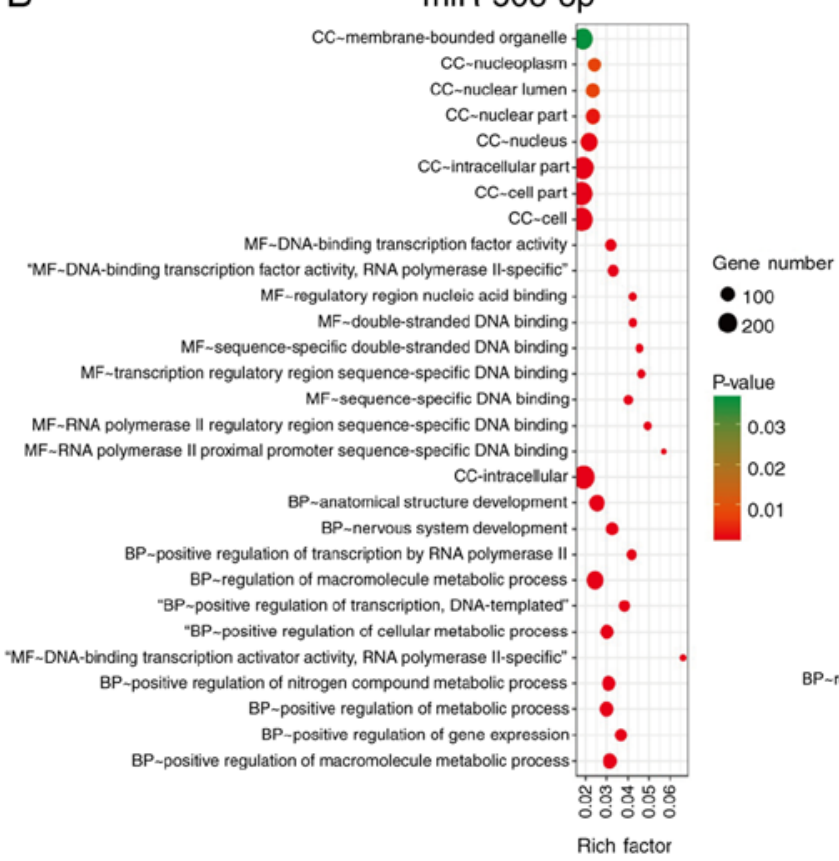

C

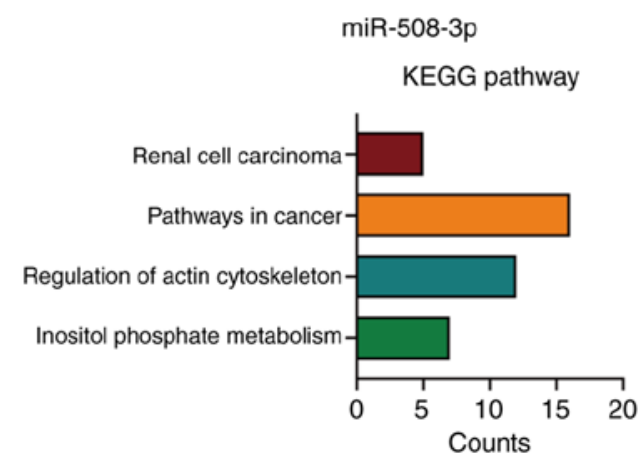

miR-885-5p

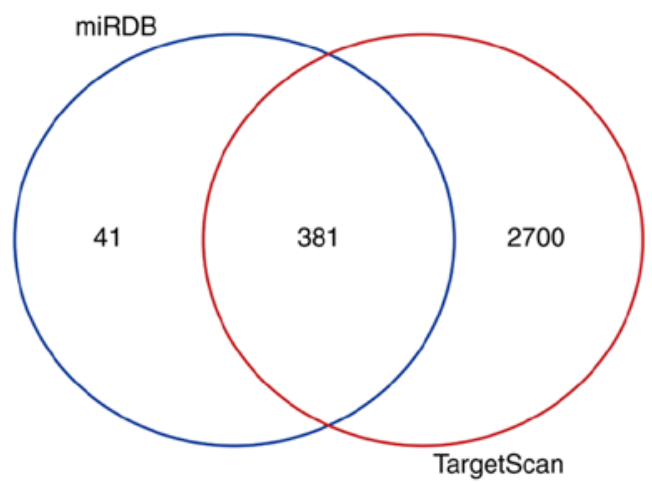

$\operatorname{miR}-885-5 p$

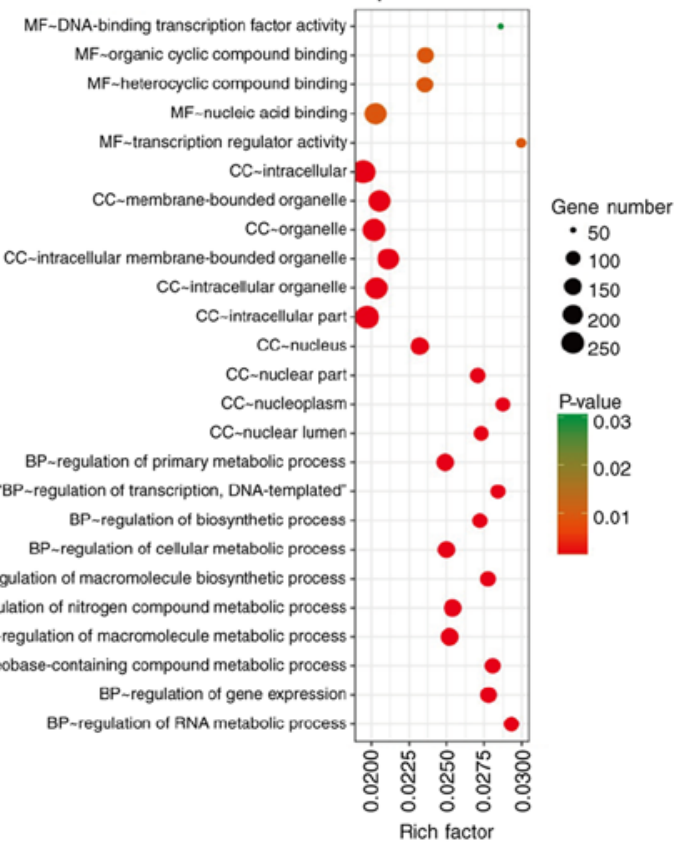

miR-885-5p

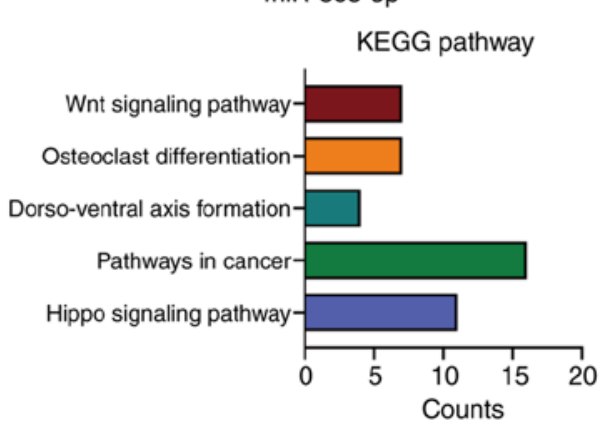

Figure 5. Functional enrichment analysis of miR-508-3p and miR-885-5p. (A) Potential targets of miR-508-3p and miR-885-5p as identified by integrated analysis of public bioinformatic algorithms TargetScan and miRDB. (B) Functional enrichment analysis of targets of miR-508-3p and miR-885-5p. (C) KEGG pathway analysis of the targets of miR-508-3p and miR-885-5p. MF, molecular functions; BP, biological processes; CC, cellular components; KEGG, Kyoto Encyclopedia of Genes and Genomes.

several signaling pathways, such as Hippo and Wnt signaling pathways. Thus, our data suggests a novel therapeutic target for ccRCC.

There are several shortcomings to our study. First, the sample sizes utilized in this study were relatively small. Second, candidate miRNAs were selected from the integrated analysis of GEO datasets and TCGA database, which may have ignored other miRNAs that act as promising diagnostic biomarkers. Finally, although we discovered miR-508-3p and miR-885-5p are associated with cellular metabolic process based on bioinformatic analysis, experiments in vitro and in vivo are still needed to validate this hypothesis.

In conclusion, this is the first time that miR-508-3p and miR-885-5p were found to be significantly dysregulated in 
ccRCC tissues and the serum of ccRCC patients. Dysregulated serum miR-508-3p and miR-885-5p was able to differentiate ccRCC from healthy controls and is associated with cellular metabolism indicating novel potential diagnostic biomarkers and therapeutic strategies for ccRCC.

\section{Acknowledgements}

Not applicable.

\section{Funding}

The present study was supported by a grant from the ZhuanBing of Shanghai Pudong New Area of Health and Family Planning Commission (grant no. PWZzb2017-17).

\section{Availability of data and materials}

The microarray data GSE109368 and GSE116251 (31) were downloaded from the GEO database in NCBI (http://www.ncbi. nlm.nih.gov/geo/). The sequencing data of miRNAs in ccRCC were downloaded from TCGA database (https://cancergenome.nih.gov/).

\section{Authors' contributions}

JZ designed the original study and drafted the manuscript. The bioinformatic analysis and the statistical analysis were carried out by SL. SL and XD collected the samples and conducted the RT-qPCR for the miRNAs. All authors edited, read and approved the manuscript and agree to be accountable for all aspects of the research in ensuring that the accuracy or integrity of any part of the work are appropriately investigated and resolved.

\section{Ethics approval and consent to participate}

This study was approved by the Research and Ethics Committee of Shanghai Putuo Liqun District Hospital. Written informed consent was obtained from all patients and healthy controls.

\section{Patient consent for publication}

Not applicable.

\section{Competing interests}

The authors declare that they have no competing interests.

\section{References}

1. Siegel RL, Miller KD and Jemal A: Cancer statistics, 2018. CA Cancer J Clin 68: 7-30, 2018.

2. Bray F, Ferlay J, Soerjomataram I, Siegel RL, Torre LA and Jemal A: Global cancer statistics 2018: GLOBOCAN estimates of incidence and mortality worldwide for 36 cancers in 185 countries. CA Cancer J Clin 68: 394-424, 2018.

3. Bhatt JR and Finelli A: Landmarks in the diagnosis and treatment of renal cell carcinoma. Nat Rev Urol 11: 517-525, 2014

4. White NM, Khella HW, Grigull J, Adzovic S, Youssef YM, Honey RJ, Stewart R, Pace KT, Bjarnason GA, Jewett MA, et al: miRNA profiling in metastatic renal cell carcinoma reveals a tumour-suppressor effect for miR-215. Br J Cancer 105: 1741-1749, 2011.
5. Crispen PL, Breau RH, Allmer C, Lohse CM, Cheville JC, Leibovich BC and Blute ML: Lymph node dissection at the time of radical nephrectomy for high-risk clear cell renal cell carcinoma: Indications and recommendations for surgical templates. Eur Urol 59: 18-23, 2011.

6. Rini BI, Campbell SC and Escudier B: Renal cell carcinoma. Lancet 373: 1119-1132, 2009.

7. Lovat F, Valeri N and Croce CM: MicroRNAs in the pathogenesis of cancer. Semin Oncol 38: 724-733, 2011.

8. Jiang L, Lv L, Liu X, Jiang X, Yin Q, Hao Y and Xiao L: MiR-223 promotes oral squamous cell carcinoma proliferation and migration by regulating FBXW7. Cancer Biomark 24: 325-334, 2019.

9. Liu X, Wang J and Zhang G: miR-4458 regulates cell proliferation and apoptosis through targeting SOCS1 in triple-negative breast cancer. J Cell Biochem 120: 12943-12948, 2019.

10. Kanlikilicer P, Bayraktar R, Denizli M, Rashed MH, Ivan C, Aslan B, Mitra R, Karagoz K, Bayraktar E, Zhang X, et al: Exosomal miRNA confers chemo resistance via targeting Cav1/p-gp/M2-type macrophage axis in ovarian cancer. EBioMedicine 38: 100-112, 2018.

11. Liu X, Xu T, Hu X, Chen X, Zeng K, Sun L and Wang S: Elevated circulating miR-182 acts as a diagnostic biomarker for early colorectal cancer. Cancer Manag Res 10: 857-865, 2018.

12. Mitchell PS, Parkin RK, Kroh EM, Fritz BR, Wyman SK, Pogosova-Agadjanyan EL, Peterson A, Noteboom J, O'Briant KC, Allen A, et al: Circulating microRNAs as stable blood-based markers for cancer detection. Proc Natl Acad Sci USA 105: 10513-10518, 2008.

13. Emami SS, Nekouian R, Akbari A, Faraji A, Abbasi V and Agah S: Evaluation of circulating miR-21 and miR-222 as diagnostic biomarkers for gastric cancer. J Cancer Res Ther 15: 115-119, 2019.

14. Sun Y, Yang B, Lin M, Yu H, Chen H and Zhang Z: Identification of serum miR-30a-5p as a diagnostic and prognostic biomarker in colorectal cancer. Cancer Biomark 24: 299-305, 2019.

15. Butz H, Nofech-Mozes R, Ding Q, Khella HWZ, Szabó PM, Jewett M, Finelli A, Lee J, Ordon M, Stewart R, et al: Exosomal MicroRNAs are diagnostic biomarkers and can mediate cell-cell communication in renal cell carcinoma. Eur Urol Focus 2: 210-218, 2016.

16. Lou N, Ruan AM, Qiu B, Bao L, Xu YC, Zhao Y, Sun RL, Zhang ST, Xu GH, Ruan HL, et al: miR-144-3p as a novel plasma diagnostic biomarker for clear cell renal cell carcinoma. Urol Oncol 35: 36.e7-e36.e14, 2017.

17. Chen X, Lou N, Ruan A, Qiu B, Yan Y, Wang X, Du Q, Ruan H, Han W, Wei H, et al: miR-224/miR-141 ratio as a novel diagnostic biomarker in renal cell carcinoma. Oncol Lett 16: 1666-1674, 2018.

18. Livak KJ and Schmittgen TD: Analysis of relative gene expression data using real-time quantitative PCR and the 2(-Delta Delta C(T)) method. Methods 25: 402-408, 2001.

19. Dai M, Li L and Qin X: Clinical value of miRNA-122 in the diagnosis and prognosis of various types of cancer. Oncol Lett 17: 3919-3929, 2019.

20. Fang H, Liu Y, He Y, Jiang Y, Wei Y, Liu H, Gong Y and An G: Extracellular vesicle-delivered miR5055p, as a diagnostic biomarker of early lung adenocarcinoma, inhibits cell apoptosis by targeting TP53AIP1. Int J Oncol 45: 1821-1832, 2019.

21. Moya L, Meijer J, Schubert S, Matin F and Batra J: Assessment of miR-98-5p, miR-152-3p, miR-326 and miR-4289 expression as biomarker for prostate cancer diagnosis. Int J Mol Sci 20: pii: E1154, 2019.

22. Tan HY, Zheng YB and Liu J: Serum miR-199a as a potential diagnostic biomarker for detection of colorectal cancer. Eur Rev Med Pharmacol Sci 22: 8657-8663, 2018.

23. Guo J, Liu C, Wang W, Liu Y, He H, Chen C, Xiang R and Luo Y: Identification of serum miR-1915-3p and miR-455-3p as biomarkers for breast cancer. PLoS One 13: e0200716, 2018.

24. Guo SJ, Zeng HX, Huang P, Wang S, Xie CH and Li SJ: MiR-508-3p inhibits cell invasion and epithelial-mesenchymal transition by targeting ZEB1 in triple-negative breast cancer. Eur Rev Med Pharmacol Sci 22: 6379-6385, 2018.

25. Huang T, Kang W, Zhang B, Wu F, Dong Y, Tong JH, Yang W, Zhou Y, Zhang L, Cheng AS, et al: miR-508-3p concordantly silences NFKB1 and RELA to inactivate canonical NF- $\mathrm{KB}$ signaling in gastric carcinogenesis. Mol Cancer 15: 9, 2016.

26. Zhai Q, Zhou L, Zhao C, Wan J, Yu Z, Guo X, Qin J, Chen J and $\mathrm{Lu} \mathrm{R}$ : Identification of miR-508-3p and miR-509-3p that are associated with cell invasion and migration and involved in the apoptosis of renal cell carcinoma. Biochem Biophys Res Commun 419: 621-626, 2012. 
27. Yu FQ, Wang Z, Wang XW, Wang SL, Li XD, Huang QS and Lin JH: MicroRNA-885-5p promotes osteosarcoma proliferation and migration by downregulation of cell division cycle protein 73 homolog expression. Oncol Lett 17: 1565-1572, 2019.

28. Dickerman BA, Torfadottir JE, Valdimarsdottir UA, Wilson KM, Steingrimsdottir L, Aspelund T, Batista JL, Fall K, Giovannucci E, Sigurdardottir LG, et al: Midlife metabolic factors and prostate cancer risk in later life. Int J Cancer 142: 1166-1173, 2018.

29. Tan W, Zhong Z, Wang S, Liu H, Yu H, Tan R, Hu X, Pan T and Wang Y: The typical metabolic modifiers conferring improvement in cancer resistance. Curr Med Chem 24: 3698-3710, 2017.

30. Rinaldi G, Rossi M and Fendt SM: Metabolic interactions in cancer: Cellular metabolism at the interface between the microenvironment, the cancer cell phenotype and the epigenetic landscape. Wiley Interdiscip Rev Syst Biol Med: Aug 30, 20172 doi: doi.org/10.1002/wsbm.1397.
31. Zhang J, Ye Y, Chang DW, Lin SH, Huang M, Tannir NM, Matin S, Karam JA, Wood CG, Chen ZN and Wu X: Global and targeted miRNA expression profiling in clear cell renal cell carcinoma tissues potentially links miR-155-5p and miR-210-3p to both tumorigenesis and recurrence. Am J Pathol 188: 2487-2496, 2018.

(i)(9) This work is licensed under a Creative Commons Attribution-NonCommercial-NoDerivatives 4.0 International (CC BY-NC-ND 4.0) License. 